\title{
Inactivation of the prelimbic or infralimbic cortex impairs decision-making in the rat gambling task
}

\author{
Fiona D. Zeeb ${ }^{1,4}$ • P. J. J. Baarendse ${ }^{2}$ - L. J. M. J. Vanderschuren ${ }^{2,3}$ • \\ Catharine A. Winstanley ${ }^{1}$
}

Received: 20 April 2015 / Accepted: 7 September 2015 /Published online: 21 September 2015

(C) Springer-Verlag Berlin Heidelberg 2015

\begin{abstract}
Rationale Studies employing the Iowa Gambling Task (IGT) demonstrated that areas of the frontal cortex, including the ventromedial prefrontal cortex, orbitofrontal cortex (OFC), dorsolateral prefrontal cortex, and anterior cingulate cortex (ACC), are involved in the decision-making process. However, the precise role of these regions in maintaining optimal choice is not clear.

Objectives We used the rat gambling task (rGT), a rodent analogue of the IGT, to determine whether inactivation of or altered dopamine signalling within discrete cortical subregions disrupts decision-making.

Methods Following training on the rGT, animals were implanted with guide cannulae aimed at the prelimbic (PrL) or infralimbic (IL) cortices, the OFC, or the ACC. Prior to testing, rats received an infusion of saline or a combination of baclofen and muscimol $(0.125 \mu \mathrm{g}$ of each/side) to inactivate
\end{abstract}

Fiona D. Zeeb

fiona.zeeb@camh.ca

Catharine A. Winstanley

cwinstanley@psych.ubc.ca

1 Department of Psychology, University of British Columbia, 2215 Wesbrook Mall, Vancouver, BC V6T 1Z3, Canada

2 Department of Translational Neuroscience, Brain Center Rudolf Magnus, University Medical Center Utrecht, Utrecht, The Netherlands

3 Department of Animals in Science and Society, Division of Behavioural Neuroscience, Faculty of Veterinary Medicine, Utrecht University, Utrecht, The Netherlands

4 Present address: Centre for Addiction and Mental Health, Section of Biopsychology, Campbell Family Mental Health Research Institute, Toronto, ON, Canada the region and an infusion of a dopamine $\mathrm{D} 2$ receptor antagonist $(0,0.1,0.3$, and $1.0 \mu \mathrm{g} /$ side).

Results Rats tended to increase their choice of a disadvantageous option and decrease their choice of the optimal option following inactivation of either the IL or PrL cortex. In contrast, OFC or ACC inactivation did not affect decision-making. Infusion of a dopamine D2 receptor antagonist into any sub-region did not alter choice preference.

Conclusions Online activity of the IL or PrL cortex is important for maintaining an optimal decision-making strategy, but optimal performance on the rGT does not require frontal cortex dopamine D2 receptor activation. Additionally, these results demonstrate that the roles of different cortical regions in cost-benefit decision-making may be dissociated using the rGT.

Keywords Anterior cingulate cortex · Decision-making · Dopamine receptor $\cdot$ Infralimbic cortex - Orbitofrontal cortex · Prelimbic cortex $\cdot$ Rat gambling task $\cdot$ Reward $\cdot$ Punishment . Probability

\section{Introduction}

An important factor in the decision-making process is the uncertain possibility of positive or negative consequences of a course of action. For this reason, the Iowa Gambling Task (IGT; Bechara et al. 1994) is frequently used to assess "realworld" decision-making. During the IGT, subjects select cards from four different decks; each card drawn results in an overall gain or loss of hypothetical money. To maximize gain, subjects must choose more often from advantageous decks - associated with smaller immediate gains but also lower penalties - and avoid the tempting, but disadvantageous decks that yield greater immediate gain but disproportionately larger 
long-term loss. Suboptimal decision-making on the IGT has been observed in numerous psychiatric disorders, including substance abuse, schizophrenia, depression, and pathological gambling (Bechara et al. 2001; Shurman et al. 2005; Goudriaan et al. 2005; Smoski et al. 2008; Cella et al. 2010).

Imaging and lesion studies have strongly implicated the involvement of frontal cortical structures, including the ventromedial prefrontal cortex (vmPFC), dorsolateral PFC (dlPFC), and cingulate cortex in IGT performance (Bechara et al. 1996, 1999, 2000; Manes et al. 2002; Ernst et al. 2002; Clark et al. 2003; Bolla et al. 2003; Fellows and Farah 2005; Naccache et al. 2005; Windmann et al. 2006; Lawrence et al. 2009; Li et al. 2010; Njomboro et al. 2012). However, the precise role of these cortical sub-regions in decision-making is still not clear, and discrepancies between studies exist. Such conflicting results in studies that include lesion patients could be due to differences in lesion size, anatomical boundaries, or duration of damage (for example, Bechara et al. 2000; Clark et al. 2003). Additionally, although imaging studies can inform which brain regions are active during performance of the IGT, these studies are unable to provide causal evidence that would determine whether a specific region is required for subjects to make optimal decisions.

Animal models may provide greater insight into the role of these cortical sub-regions in decision-making. Much tighter control is possible over the location and implementation of neuronal silencing, and causal roles for different brain regions are easier to establish. The rat gambling task (rGT) developed by Zeeb et al. (2009) is a complex decision-making task comparable to the IGT. Similar to the IGT, options associated with smaller immediate gains but less long-term loss are advantageous, whereas disadvantageous options are associated with larger immediate gain but much greater loss.

Damage to the vmPFC in humans - encompassing the OFC — caused subjects to choose the disadvantageous decks more often on the IGT compared to healthy controls (Bechara et al. 1994, 1999). Additionally, greater activity in the OFC has also been associated with improved IGT performance (Bolla et al. 2003). However, increased activity was detected in cortical regions including the cingulate cortex and OFC while subjects performed the IGT but failed to prefer the advantageous decks for nearly half of the IGT or variants of the IGT (Li et al. 2010). Interestingly, rats with orbitofrontal cortex (OFC) lesions developed the optimal strategy on the rGT (Zeeb and Winstanley 2011). The OFC has also been implicated in reversal learning and perseveration, and a large number of rats with OFC lesions demonstrated an inflexible pattern of decision-making (Dalley et al. 2004; Cardinal 2006; Boulougouris et al. 2007; Rivalan et al. 2011). These findings suggest that OFC damage may not increase risky decisionmaking per se, but increase perseveration or disrupt reversal learning, which may manifest as increased risky choice on the IGT (Fellows and Farah 2005).
The anterior cingulate cortex (ACC) is another important region in cost-benefit decision-making. On the IGT, subjects with ACC damage demonstrated increased choice of the disadvantageous options (Naccache et al. 2005; Njomboro et al. 2012). Interestingly, increased activation of the ACC was observed following too safe or too risky choices on a gambling task (Hewig et al. 2009). Therefore, the ACC may be required for subjects to learn the contingencies of winning and losing, but not the actual decision-making process, similar to the role of the OFC.

The dIPFC of humans, homologous to the medial prefrontal cortex (mPFC) in rats (Uylings et al. 2003; Seamans et al. 2008), is largely involved in working memory (Ragozzino 2007). However, the human dlPFC and areas of the rat $\mathrm{mPFC}$ - specifically the prelimbic (PrL) and infralimbic (IL) sub-regions - may also be important in cost-benefit decisionmaking. Humans with dIPFC damage prefer the disadvantageous decks more than control subjects on the IGT (Fellows and Farah 2005), and rats with combined lesions of the IL and PrL cortices chose the advantageous options less than control rats on the rGT (Paine et al. 2013). Although the PrL and IL cortices share some similarities, these cortical regions have different anatomical projections (Vertes 2004; Hoover and Vertes 2007) and therefore could make distinct and independent contributions to decision-making.

In sum, although specific sub-regions of the frontal cortex are clearly involved in decision-making, the precise role of these regions is diverse. However, all regions have been implicated in performance of a complex cost-benefit decisionmaking task, i.e., the IGT. Therefore, the present study determined whether acute inactivation of each region disrupted decision-making using the rGT once a stable preference had been established. Additionally, as a systemic injection of a dopamine (DA) D2 receptor antagonist enhanced choice of the optimal option on the rGT (Zeeb et al. 2009, 2013), we also determined whether direct antagonism of the DA D2 receptors in each sub-region would likewise affect choice preference.

\section{Methods}

\section{Subjects}

Subjects were 40 male Long Evans rats (Charles River Laboratories, St. Constant, QC, Canada) and 16 male Lister Hooded rats (Harlan, Horst, The Netherlands), weighing approximately $300 \mathrm{~g}$ at the start of the experiment. Both Long Evans and Lister Hooded rats are outbred hooded rat strains and follow a similar growth curve. Animals were initially housed in pairs under a 12-h light cycle. Following surgery, animals were singly housed. Water was available ad libitum. Rats received free access to food until 1 week prior to the start 
of behavioural testing, at which point animals were gradually restricted to approximately $14 \mathrm{~g}$ of standard laboratory rat chow per day, available immediately after testing to maintain animals at approximately $85-90 \%$ of their free-feeding weight. For Long Evans rats, testing occurred at the University of British Columbia; testing of Lister Hooded rats occurred at the University Medical Center Utrecht. Experimental protocols were approved by the Animal Care Committee of the University of British Columbia in accordance with the Canadian Council of Animal Care or the Animal Ethics Committee of Utrecht University in accordance with Dutch laws (Wet op de Dierproeven 1996) and European regulations (Guideline 86/609/EEC).

\section{Rat gambling task}

Apparatus Descriptions of the testing chambers and protocols for the rGT have been provided in previous reports (see Zeeb et al. 2009; Baarendse et al. 2013). Briefly, rGT testing took place in standard 5-hole operant conditioning chambers (Med Associates, St. Albans, VT, USA) fitted with an array of five response holes located on one wall and a food tray on the opposite wall, $2 \mathrm{~cm}$ above a metal bar floor. A stimulus light was located within each hole, and a horizontal infrared beam detected nose-poke responses into these apertures. The entire chamber could be illuminated by a house light located at the top of the chamber. Dustless precision food pellets $(45 \mathrm{mg}$, Bioserv, Frenchtown, NJ, USA) could be delivered into the food tray from an external pellet dispenser. Chambers were controlled by software written in Med PC running on an IBMcompatible computer.

Training Rats received two 30 min habituation sessions, during which the chambers were turned on and pellets were placed in the response holes and food tray. Animals were next trained to make a nosepoke response into an illuminated response hole within $10 \mathrm{~s}$ for a single pellet reward. Once rats were completing these sessions with at least $80 \%$ of trials correct and less than $20 \%$ omissions (6-8 sessions), animals received seven forced choice sessions (see Zeeb et al. 2009). Animals were then tested on the rGT for the duration of the experiment.

rGT Animals were tested once daily in a single 30 min session. The reinforcement schedule of each option is depicted in Table 1 and is identical to previous reports (Zeeb et al. 2009). Long Evans rats initiated a trial by making a nosepoke response into the illuminated food tray, and this response extinguished the tray light signaling the start of a $5 \mathrm{~s}$ intertrial interval (ITI). Following the ITI, four holes (holes 1, 2, 4, and 5 ) were illuminated for $10 \mathrm{~s}$, and a nosepoke response into any of these holes immediately extinguished all stimulus lights. If the trial was rewarded, the traylight turned on and the
Table 1 Possible outcomes following a win or a loss on the rGT

\begin{tabular}{llllll}
\hline & P1 & P2 & P3 & P4 \\
\hline Chance of a win trial & $90 \%$ & $80 \%$ & $50 \%$ & $40 \%$ \\
Number of pellets rewarded (win trial only) & 1 & 2 & 3 & 4 \\
Duration of Time out (s) (loss trial only) & 5 & 10 & 30 & 40 \\
\hline
\end{tabular}

corresponding number of food pellets were immediately delivered. Collection of this reward initiated the next trial for Long Evans rats. For Lister Hooded rats, the next trial began after $5 \mathrm{~s}$ as the food tray in the testing chamber was not equipped with an infrared detector. If the trial was not rewarded, the stimulus light within the chosen hole flashed at $0.5 \mathrm{~Hz}$ for the duration of the corresponding time-out period. Perseverative responses made at the array or food tray during the time-out period or following reward delivery were recorded, but had no scheduled consequences. If the animal failed to make a response in $10 \mathrm{~s}$ all stimulus lights were extinguished, after which a next trial either began (Lister Hooded rats) or the traylight was re-illuminated allowing the animal to start the next trial (Long Evans rats). A nosepoke response into any of the holes at the array during the ITI was classified as a premature response - a measure of impulsive action similar to premature responses recorded on the 5-choice serial reaction time task (Robbins 2002). Premature responses were punished with the illumination of the house light for $5 \mathrm{~s}$, after which a new trial began or could be initiated.

The optimal choice in the rGT is the two-pellet option (P2), as this option results in the most reward earned per unit time due to the design of the reinforcement schedules (Zeeb et al. 2009). The next best option is $P 1$, and the two disadvantageous options are $\mathrm{P} 3$ and $\mathrm{P} 4$ due to the lower probability of receiving reward and the longer time-out periods that could occur. Two versions of the rGT were used which differed only in the spatial location of the options and were counterbalanced across all animals (Zeeb et al. 2009). Animals were trained once daily, 5-6 days per week, on the rGT until stable baseline behaviour was observed (minimum of 24 sessions).

\section{Surgery}

Long Evans rats were anesthetized with ketamine (Ketaset, 100 mg/kg i.m.; Vetoquinol, Lavaltrie, QC, Canada) and xylazine (Rompun, $10 \mathrm{mg} / \mathrm{kg}$ i.m.; UBC Animal Care Centre, Vancouver, BC, Canada). Lister Hooded rats were anesthetized with ketamine (Narketan, $75 \mathrm{mg} / \mathrm{kg}$ i.m., Vetoquinol, 's Hertogenbosch, The Netherlands) and medetomidine (Seda-start, $0.4 \mathrm{mg} / \mathrm{kg}$ s.c., Astfarma, Oudewater, The Netherlands). Next, rats were secured into a stereotaxic frame (David Kopf Instruments, Tujunga, CA, USA) in a flat skull position. Bilateral 22-gauge, stainless steel cannulae (Plastics One, Roanoke, VA, USA) were then 
implanted and secured to the skull using stainless steel screws and dental cement. The stereotaxic coordinates (in $\mathrm{mm}$ ) used were: $\mathrm{OFC}$ site, anterior-posterior $(\mathrm{AP})+3.8$, medial-lateral $(\mathrm{ML}) \pm 2.6$, dorsal-ventral (DV) -2.9 ; ACC site, AP +2.2, $\mathrm{ML} \pm 0.7, \mathrm{DV}-0.8$, IL site, $\mathrm{AP}+2.7, \mathrm{ML} \pm 0.6, \mathrm{DV}-3.4$; PrL site, AP +3.0, ML $\pm 0.7, \mathrm{DV}-2.2$ (Paxinos and Watson 1998). The AP coordinate was taken from bregma, the ML coordinate was taken from the midline, and the DV coordinate was taken from dura. Obdurators flush with the end of the guide cannula were then inserted and secured with plastic dust caps (Plastics One, Roanoke, VA, USA). Cannulae for Long Evans rats were aimed at the OFC, ACC, or PrL region; cannulae for Lister Hooded rats were aimed at the IL cortex. Animals recovered for 1 week in their home cage, after which rGT testing resumed.

\section{Microinfusion procedure}

Once animals established stable post-operative performance on the rGT (8-13 sessions), rats were habituated to the infusion procedure with two mock infusions. Methods for microinfusions were similar to previously published reports (Veeneman et al. 2012; Zeeb et al. 2010). Briefly, rats were gently restrained while dust caps and obdurators were removed. A 28-gauge injector extending $1 \mathrm{~mm}$ (OFC, IL) or $1.5 \mathrm{~mm}$ (ACC, PrL) below the guide cannula was then lowered into each guide. Using a dual channel infusion pump, $0.5 \mu \mathrm{L}$ of solution was infused at a rate of $0.25 \mu \mathrm{L} / \mathrm{min}$, after which the injector was left in place for $1 \mathrm{~min}$ to allow for diffusion. The injectors were then removed, and the obdurators and dust caps were replaced. Rats were placed in a holding cage similar to their home cage for $10 \mathrm{~min}$ before being moved to the operant conditioning chamber and starting the rGT.

All rats first received an infusion of sterile saline $(0.5 \mu \mathrm{L})$ or an infusion of a combination of the $\mathrm{GABA}_{\mathrm{A}}$ and $\mathrm{GABA}_{\mathrm{B}}$ receptor agonists $(0.125 \mu \mathrm{g}$ of each compound in $0.5 \mu \mathrm{L}$ of sterile saline) counterbalanced within each group. Rats then received an infusion of either sterile saline $(0.5 \mu \mathrm{L})$ or the dopamine $\mathrm{D} 2$ receptor antagonist, eticlopride. The doses of eticlopride administered were $0.1,0.3$, and $1.0 \mu \mathrm{g} /$ side dissolved in $0.5 \mu \mathrm{L}$ of sterile saline. The order of these infusions was counterbalanced according to a diagram-based Latin Square design. Infusion test days were separated by at least $72 \mathrm{~h}$. Due to the blocked cannulae, one animal with cannulae aimed at the OFC and one animal with cannulae aimed at the PrL were removed from the study after receiving the first two infusions.

Baclofen hydrochloride, muscimol hydrobromide, and eticlopride hydrochloride were purchased from SigmaAldrich (Oakville, ON, Canada) or Tocris Bioscience
(Abingdon, UK) and freshly prepared on each microinfusion day. Doses were calculated according to the salt.

\section{Data analyses}

Repeated-measures analysis of variance (ANOVA) and post hoc analyses were conducted using SYSTAT for Windows (version 12.00.08; SSI). The percent choice of each option (of total number of trials completed), percentage of premature responses made (of total number of trials initiated), number of trials completed, number of trials omitted, total number of perseverative responses made following a rewarded or punished trial, and the latency to make a choice were analyzed. Similar to previous studies (for example, Zeeb et al. 2009), variables calculated as a percentage were subjected to an arcsine transformation prior to statistical analysis to limit the effect of the artificially imposed ceiling (McDonald 2009). To determine baseline stability, a repeated-measures ANOVA was conducted with version (2 levels; A or B) as a betweensubject factor and session (3 levels) and choice (4 levels; P1-4) as within-subject factors. Stability was determined by a nonsignificant effect of session and session $\times$ choice (Zeeb et al. 2009; Zeeb and Winstanley 2011). Similar to previous studies (Zeeb et al. 2009; Zeeb and Winstanley 2011; Baarendse et al. 2013), the rGT version used did not differentially affect decision-making; there was no significant effect of version, version $\times$ site, version $\times$ choice, or version $\times$ choice $\times$ site (all F's $<1.5$ ) across the last three baseline sessions. Therefore, data from animals tested on each version were combined for analyses.

Data from the microinfusions were analyzed according to a repeated-measures ANOVA with dose (2 levels: saline vs inactivation; 4 levels: saline vs eticlopride doses) and, when appropriate, choice (4 levels; P1-4) as within-subject factors. Data from the first saline infusion was compared to the baclofen and muscimol cocktail infusion (inactivation); data collected following the second saline infusion was used in the analyses following eticlopride infusions. Infusion site (4 levels) was used as a between-subject factor to determine whether animals' rGT performance differed between the different group of rats following a saline infusion. Separate analyses were conducted for each group following the drug infusions. If the outcome of the ANOVA was significant at the level of dose $\times$ choice, post hoc analyses using $t$ tests were conducted comparing each dose of drug to the corresponding saline infusion for each measurement. A significance level of $p \leq 0.05$ was used for all analysis.

\section{Histology}

Following completion of the final infusion test day, animals were sacrificed by exposure to an increasing concentration of carbon dioxide. Brains were then removed and post-fixed in a 
solution of $4 \%$ formaldehyde in phosphate buffered saline for at least $24 \mathrm{~h}$. Coronal sections $(40 \mu \mathrm{m})$ throughout the area of interest were taken using a cryostat, mounted on gel-coated slides, and stained with cresyl violet. The location of the infusion sites were mapped onto standardized images of the rat brain (Paxinos and Watson 1998).

\section{Results}

\section{Cannulae placements}

The locations of the infusion site for all regions are presented in Fig. 1. Animals with placements outside the target regions were excluded. Ten animals had placements in the OFC (version $\mathrm{A} n=6$, version $\mathrm{B} n=4$ ) and $\mathrm{ACC}$ (version A $n=6$, version B $n=4$ ), seven rats had placements in the IL (version A $n=3$, version $\mathrm{B} n=4$ ), and 11 rats had placements in the PrL (version $\mathrm{A} n=5$, version $\mathrm{B} n=6$ ).

\section{a}

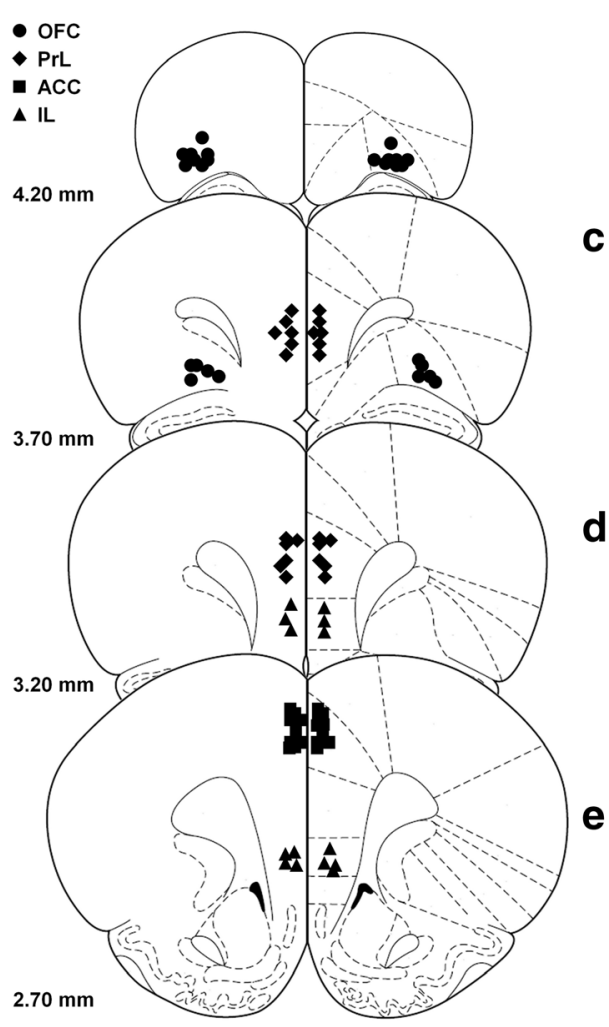

b

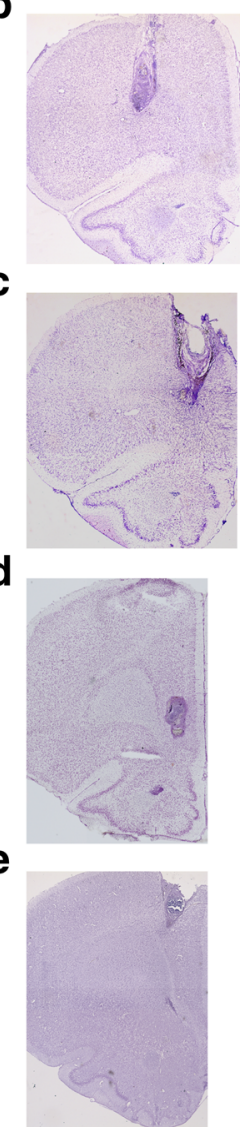

Fig. 1 Verification of cannulae placements. A schematic depiction of the location of the infusion site for rats with cannuale aimed at the OFC (circles), PrL cortex (diamonds), ACC (squares), and IL cortex (triangles) are shown (a). Photomicrographs of a representative sample from rats with placements in the OFC (b), PrL cortex (c), IL cortex (d), and ACC (e) are also shown. Only rats with correct placements were included in the study. Distances from bregma for each image are shown in millimeters. Images adapted from Paxinos and Watson (1998)

\section{Inactivation results}

Choice Analysis of data from the saline infusion demonstrated there were no differences in choice preference between all groups. Although inactivation of the OFC did not significantly alter choice preference, inactivation of the PrL cortex and ACC tended to change the pattern of choice (Fig. 2; ACC: dose: $\mathrm{F}_{1,9}=4.837, p=0.06$; PrL: dose: $\mathrm{F}_{1,10}=4.222, p=0.07$ ). Additionally, inactivation of the IL cortex impaired the ability of animals to choose optimally on the rGT (Fig. 2; dose: $\mathrm{F}_{1,6}=$ 9.551, $p=0.02$; dose $\times$ choice: $\mathrm{F}_{3,18}=2.493, p=0.09$ ). Visual inspection of the data suggest choice preference did not largely change following inactivation of the ACC, whereas inactivation of the PrL cortex decreased choice of $\mathrm{P} 2$ and increased choice of P4, the worst option. Likewise, compared to saline, inactivation of the IL cortex appeared to decrease choice of P2 - the optimal option - and increase choice of P4.

As a combined lesion of the PrL and IL cortex increased risky decision-making on the rGT (Paine et al. 2013) and there were visually similar effects of the inactivation of the IL and PrL cortices, here, we combined data from the IL and PrL groups for an exploratory analysis. There was no effect of site, dose $\times$ site, choice $\times$ site,
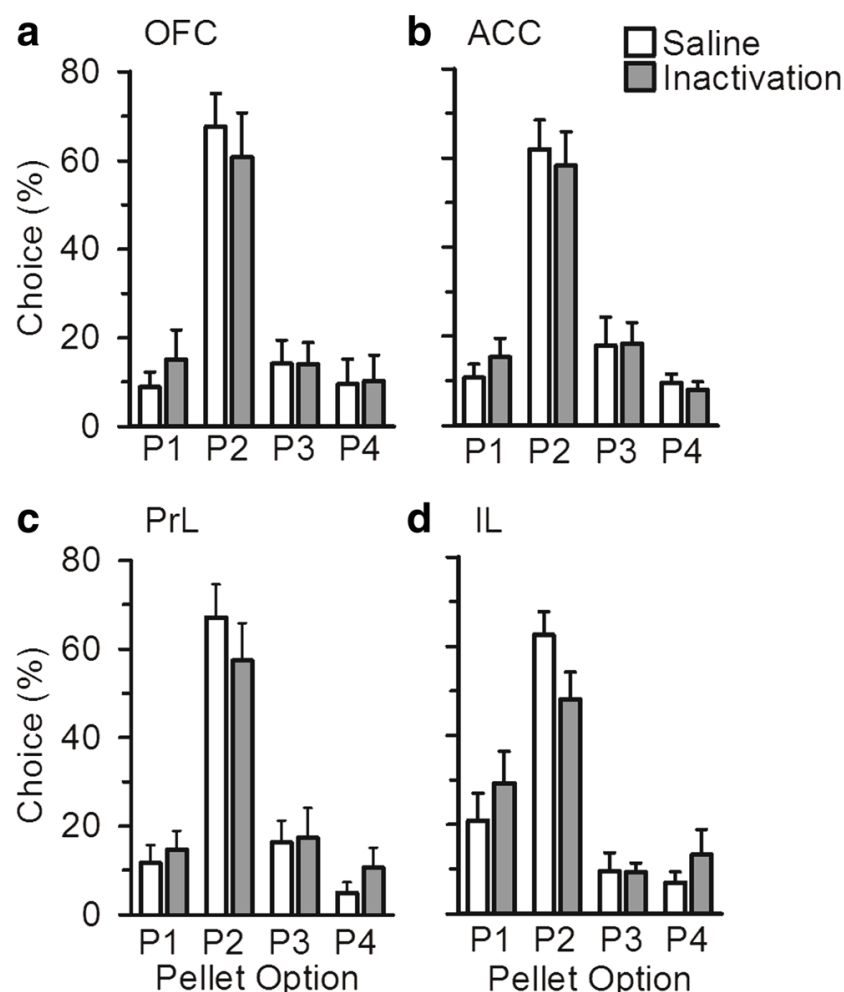

Fig. 2 Effects of an inactivation of different cortical sub-regions on choice preference in the rGT. Inactivation of the OFC (a) or ACC (b) did not alter choice preference in the rGT. However, visual inspection of the data demonstrated that inactivation of the PrL cortex caused rats to choose the worst option, P4, more, and possibly P2 less (c). Likewise, inactivation of the IL cortex (d) appeared to cause animals to choose P4 more and $\mathrm{P} 2$ less 
or dose $\times$ choice $\times$ site, demonstrating there were no differences between these groups. However, there was a significant effect of dose $\left(\mathrm{F}_{1,16}=10.173, p=0.006\right)$ and a significant interaction of dose $\times$ choice $\left(\mathrm{F}_{3,48}=4.566, p=0.007\right)$. Post hoc analyses only revealed a significant decrease in choice of P2 $(\mathrm{t}(17)=2.780, p=0.01)$ and increase in choice of P4 ( $\mathrm{t}(17)=-2.952 p=0.009)$. Together, these data suggest that inactivation of the PrL and IL cortex may similarly disrupt decision-making on the rGT; however, the groups used in the present study may have been underpowered to detect a significant difference when analyzed alone.

Other variables Data for the other variables measured during rGT performance and the outcome of any post hoc analyses performed are presented in Table 2. All rats completed a similar number of trials following a saline infusion or an inactivation, and there were no differences in the number of premature responses made between groups or following the inactivation. Although animals with cannulae aimed at the IL cortex omitted more trials than the other groups (infusion site: $\mathrm{F}_{3,34}=101.769, p<0.001$ ), compared to saline, inactivation of any region did not significantly increase the number of trials omitted.

The latency to choose an option was greatest for rats with cannulae aimed at the IL cortex (infusion site: $\mathrm{F}_{3,34}=6.892$, $p=0.001$ ). However, compared to a saline infusion, post hoc analyses revealed that choice latency increased following inactivation of the OFC, ACC, or PrL cortex, but not the IL cortex (dose: $\mathrm{F}_{1,34}=25.500, p<0.001$ ). Additionally, the number of perseverative responses made was smallest for rats with cannulae aimed at the IL cortex and tended to increase in all
Table 2 Other variables measured during rGT performance

\begin{tabular}{|c|c|c|c|c|c|}
\hline \multirow[t]{2}{*}{ Variable } & \multirow[t]{2}{*}{ Infusion drug } & \multicolumn{4}{|l|}{ Infusion site } \\
\hline & & OFC & $\mathrm{ACC}$ & IL & PrL \\
\hline \multirow[t]{6}{*}{ Trials completed } & Saline & $97.8 \pm 10.4$ & $83.8 \pm 6.6$ & $89.0 \pm 5.1$ & $81.0 \pm 6.5$ \\
\hline & Inactivation & $94.1 \pm 11.8$ & $82.8 \pm 6.5$ & $70.3 \pm 7.5$ & $89.9 \pm 8.4$ \\
\hline & Saline & $96.9 \pm 10.1$ & $89.5 \pm 8.2$ & $71.9 \pm 4.3$ & $99.7 \pm 10.3$ \\
\hline & E $0.1 \mu \mathrm{g} /$ side & $105.3 \pm 11.8$ & $89.2 \pm 7.3$ & $73.3 \pm 5.0$ & $105.0 \pm 11.1$ \\
\hline & E $0.3 \mu \mathrm{g} /$ side & $104.0 \pm 11.7$ & $90.9 \pm 8.6$ & $68.4 \pm 4.7$ & $97.0 \pm 6.2$ \\
\hline & E $1.0 \mu \mathrm{g} / \mathrm{side}$ & $102.3 \pm 11.9$ & $101.9 \pm 8.3$ & $65.0 \pm 4.0$ & $106.3 \pm 10.7$ \\
\hline \multirow[t]{6}{*}{ Trials omitted } & Saline & $0.1 \pm 0.1$ & $0.4 \pm 0.2$ & $21.4 \pm 4.1$ & $0.2 \pm 0.1$ \\
\hline & Inactivation & $2.3 \pm 1.4$ & $0.6 \pm 0.2$ & $29.4 \pm 4.2$ & $1.1 \pm 0.5$ \\
\hline & Saline & $1.1 \pm 0.7$ & $0.1 \pm 0.1$ & $29.4 \pm 4.8$ & $0.4 \pm 0.2$ \\
\hline & E $0.1 \mu \mathrm{g} /$ side & $0.4 \pm 0.2$ & $0.2 \pm 0.1$ & $31.1 \pm 4.5$ & $0.3 \pm 0.2$ \\
\hline & E $0.3 \mu \mathrm{g} /$ side & $0.6 \pm 0.3$ & $0.0 \pm 0.0$ & $34.1 \pm 7.2$ & $0.4 \pm 0.2$ \\
\hline & E $1.0 \mu \mathrm{g} / \mathrm{side}$ & $0.8 \pm 0.5$ & $0.1 \pm 0.1$ & $35.3 \pm 4.3^{\#}$ & $0.1 \pm 0.1$ \\
\hline \multirow[t]{6}{*}{ Premature responses $(\%)$} & Saline & $30.0 \pm 5.3$ & $34.8 \pm 4.5$ & $21.9 \pm 1.9$ & $44.3 \pm 3.9$ \\
\hline & Inactivation & $31.2 \pm 5.4$ & $38.5 \pm 5.4$ & $27.5 \pm 5.1$ & $35.0 \pm 4.9$ \\
\hline & Saline & $27.0 \pm 3.4$ & $24.9 \pm 5.4$ & $27.0 \pm 3.0$ & $27.7 \pm 4.9$ \\
\hline & E $0.1 \mu \mathrm{g} /$ side & $25.2 \pm 5.7$ & $27.5 \pm 4.4$ & $25.1 \pm 3.2$ & $24.6 \pm 4.9$ \\
\hline & E $0.3 \mu \mathrm{g} / \mathrm{side}$ & $23.2 \pm 4.8$ & $26.6 \pm 4.6$ & $23.4 \pm 2.4$ & $24.6 \pm 4.0$ \\
\hline & E $1.0 \mu \mathrm{g} / \mathrm{side}$ & $22.8 \pm 4.4$ & $20.6 \pm 3.9$ & $26.0 \pm 3.4$ & $27.3 \pm 5.1$ \\
\hline \multirow[t]{6}{*}{ Choice latency (s) } & Saline & $1.0 \pm 0.1$ & $0.8 \pm 0.1$ & $1.6 \pm 0.2$ & $0.8 \pm 0.1$ \\
\hline & Inactivation & $1.6 \pm 0.2 *$ & $1.2 \pm 0.1 *$ & $1.7 \pm 0.2$ & $1.4 \pm .1 *$ \\
\hline & Saline & $0.9 \pm 0.1$ & $0.9 \pm 0.1$ & $1.9 \pm 0.3$ & $0.9 \pm 0.1$ \\
\hline & E $0.1 \mu \mathrm{g} /$ side & $0.9 \pm 0.1$ & $0.8 \pm 0.1$ & $2.1 \pm 0.3$ & $1.0 \pm 0.2$ \\
\hline & E $0.3 \mu \mathrm{g} / \mathrm{side}$ & $1.0 \pm 0.1$ & $0.8 \pm 0.1$ & $1.8 \pm 0.2$ & $1.1 \pm 0.2$ \\
\hline & E $1.0 \mu \mathrm{g} / \mathrm{side}$ & $0.9 \pm 0.1$ & $0.9 \pm 0.1$ & $1.7 \pm 0.3$ & $1.0 \pm 0.2$ \\
\hline \multirow[t]{6}{*}{ Total perseverative responses } & Saline & $127.6 \pm 15.9$ & $161.3 \pm 24.7$ & $48.4 \pm 7.3$ & $210.9 \pm 81.5$ \\
\hline & Inactivation & $166.6 \pm 26.0 *$ & $191.4 \pm 30.4$ & $67.7 \pm 9.9$ & $238.5 \pm 50.6$ \\
\hline & Saline & $105.3 \pm 15.4$ & $203.8 \pm 34.3$ & $51.4 \pm 8.1$ & $191.8 \pm 47.7$ \\
\hline & E $0.1 \mu \mathrm{g} / \mathrm{side}$ & $128.9 \pm 24.4$ & $182.5 \pm 34.2$ & $36.1 \pm 4.7$ & $190.3 \pm 68.9$ \\
\hline & E $0.3 \mu \mathrm{g} / \mathrm{side}$ & $114.8 \pm 9.2$ & $214.9 \pm 39.1$ & $40.7 \pm 10.9$ & $225.8 \pm 67.1$ \\
\hline & E $1.0 \mu \mathrm{g} /$ side & $120.3 \pm 15.9$ & $177.7 \pm 24.1$ & $40.6 \pm 6.7$ & $178.0 \pm 25.7$ \\
\hline
\end{tabular}

${ }^{*} p<0.05$ following a $t$ test compared to saline; ${ }^{*} p>0.05$ and $p<0.07$ following a $t$ test compared to saline 
groups following inactivation (infusion site: $\mathrm{F}_{3,34}=2.496, p=$ 0.08 ; dose: $\left.\mathrm{F}_{1,34}=4.305, p=0.05\right)$. However, post hoc analyses demonstrated that this effect was only significant for rats that received an OFC inactivation (OFC: $\mathrm{t}(9)=-2.326, p=$ $0.05)$.

\section{Results following microinfusions of eticlopride}

Similar to the results from the inactivation study, there were no differences in choice preference between groups following a saline infusion. Intra-cranial infusion of eticlopride at any dose did not significantly alter choice preference in any group (Fig. 3). Although animals with cannulae aimed at the IL cortex omitted more trials than the other groups (infusion site: $\mathrm{F}_{3,32}=67.987, p<0.001$ ), made less perseverative responses (infusion site: $\mathrm{F}_{3,32}=$ $4.305, p=0.01$ ), and took longer to choose an option (infusion site: $\left.\mathrm{F}_{3,32}=10.889, p<0.001\right)$, infusion of eticlopride into any region tested did not alter the other variables analyzed during rGT performance (Table 2).

\section{Discussion}

Here, we demonstrated that acute inactivation of either the PrL or IL cortices similarly disrupted established decision-making preferences using the rGT - a risky decision-making test based on the IGT involving both gains and losses. Although the effects of inactivation of the PrL or IL cortex alone did not reach significance, combined analyses of these two groups demonstrated an increased choice of $\mathrm{P} 4$, a disadvantageous option, and decreased choice of $\mathrm{P} 2$, the optimal option. These results highlight the important role for online activity of the mPFC in maintaining an optimal strategy on the rGT. In contrast, inactivation of the OFC and ACC did not alter choice patterns. Therefore, the roles of different cortical regions in cost-benefit decision-making may be dissociated using the rGT, a result difficult to discern from lesion or imaging studies that employ the IGT.

Human subjects with damage to the dIPFC choose the disadvantageous decks more often than healthy controls on the IGT (Fellows and Farah 2005). In contrast, using a single session rat IGT, the majority of animals with $\mathrm{mPFC}$ damage displayed either an inflexible pattern of decision-making (i.e., failed to sample all options prior to choosing a preferred strategy) or were undecided (i.e., failed to demonstrate a clear preference for either the advantageous or disadvantageous options) (Rivalan et al. 2011). Likewise, inactivation of the PrL cortex impaired the animals' ability to effectively alter their decision-making strategy when the reward value was altered during a probabilistic decision-making task (St Onge and Floresco 2010). As the IGT is most often used as a single
Fig. 3 Infusion of the DA D2 receptor antagonist eticlopride into the OFC (a), ACC (b), PrL cortex (c), or IL cortex (d) did not alter decision-making on the rGT
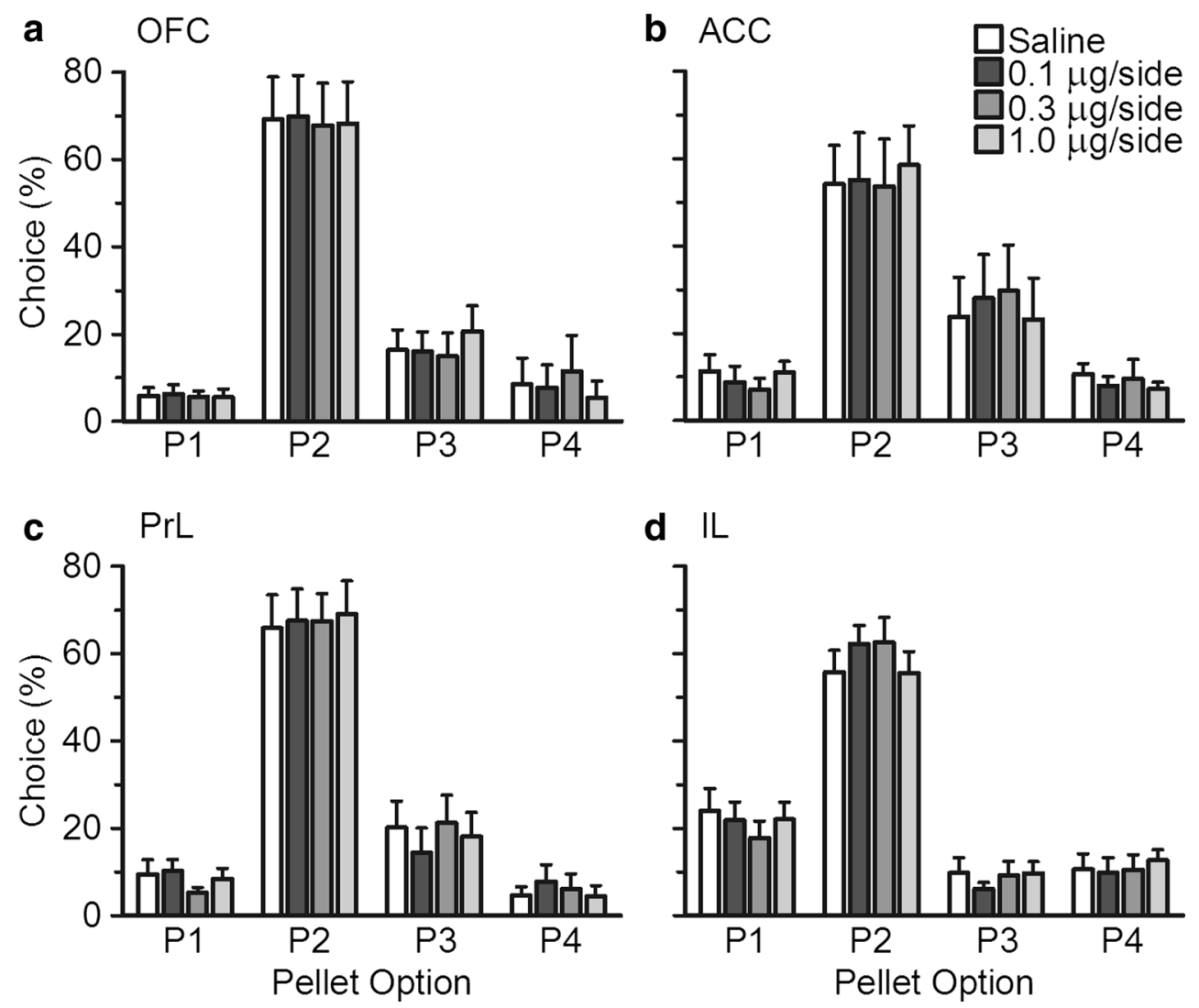
session task, similar to the rat IGT used by Rivalan and colleagues (2009), it is not clear whether the disadvantageous decision-making pattern observed in human subjects with damage to the dIPFC are due to an actual decision-making deficit or a failure to learn the task rules.

In the present study, rats were first trained to perform the rGT. Therefore, it was certain that animals had learned the optimal strategy prior to any manipulation. As increased activation of cortical regions, including the OFC and medial PFC, were observed following testing on a rat IGT (Fitoussi et al. 2014), these regions are likely active during rGT performance. We found that inactivation of the IL or PrL cortex both visually decreased choice of the optimal option and increased choice of the most disadvantageous option. Combined analyses statistically supported these observations. Interestingly, combined lesions of the IL and PrL cortices after animals had been trained on the rGT decreased advantageous decision-making compared to control rats (Paine et al. 2013), further implicating these regions in maintaining advantageous choice. Additionally, infusion of a $\mathrm{GABA}_{\mathrm{A}}$ antagonist into the PrL and IL regions also decreased choice of the optimal option on the rGT (Paine et al. 2014). Therefore, disruptions in GABA signaling within the PrL and IL regions may impact this form of decision making. In sum, results from the present experiment support findings from previous studies that suggest a role for the PrL and IL cortices in maintaining optimal choice.

Although there were strain and minor methodological differences between rats with cannulae aimed at the IL or PrL cortices, animals were exposed to the same task contingencies, made voluntarily choices, and there were no differences in choice patterns between these rats. Both strains used in the present study (Long Evans and Lister Hooded rats) have been used to examine decision-making on the rGT (for example, Zeeb et al. 2009; Zeeb and Winstanley 2011; Baarendse et al. 2013; Connolly et al. 2015), and little strain differences have been previously noted. For example, acute administration of amphetamine increased choice of P1 and decreased choice of P2 in both Long Evans (Zeeb et al. 2009, 2013) and Lister Hooded (Baarendse et al. 2013) rats. Furthermore, animals of both strains appeared to learn the rGT at a similar rate, resulting in comparable choice preferences. Additionally, similar effects following inactivation of the PrL or IL cortices support results from a previous study using Sprague Dawley rats that found lesions of the mPFC increased risky choice on the rGT (Paine et al. 2013). Therefore strain does not appear to have a significant impact on choice preferences measured on the rGT.

Premature responding - a measurement of impulsive action - on the rGT is recorded in a similar fashion to premature responding on the 5CSRTT, wherein animals are required to wait after a trial is initiated before responding in the apertures. Additionally, similarities between premature responding on the rGT and 5CSRTT have also been reported. For example, a dopamine D2 receptor antagonist did not affect premature responding, whereas amphetamine increased premature responding on both tasks (Zeeb et al. 2009; Zeeb et al. 2013; Baarendse and Vanderschuren 2012; Baarendse et al. 2013, see Robbins 2002; Pattij and Vanderschuren 2008 for reviews of the 5CSRTT literature). Inactivation of the ACC, or the IL or PrL cortex, did not alter premature responding on the rGT. These results are similar to previous findings, demonstrating that $\mathrm{mPFC}$ lesions also do not increase premature responding on the rGT (Paine et al. 2013). Additionally, IL cortex lesions did not affect impulsivity on the stop-signal reaction-time (SSRT) task which measures the ability for subjects to inhibit a response that has already been initiated (Eagle et al. 2008).

In contrast, previous studies have found that lesions of the ACC, IL cortex, or the mPFC increased premature responding on the 5 -choice serial reaction time task (5CSRTT), a test of attention and impulsive action (Muir et al. 1996; Tsutsui-Kimura et al. 2014; Chudasama et al. 2003). Likewise, inactivation of the IL (but not PrL) cortex increased premature responding on the 5CSRTT (Murphy et al. 2012). In the 5CSRTT, a light is only briefly illuminated in one aperture and animals must respond in that same aperture to obtain reward; there appears to be a definite urge to attend and respond quickly in the 5CSRTT. In contrast, on the rGT, four apertures are always illuminated for $10 \mathrm{~s}$ or until a response is made, and rats can choose any of these options. The decreased urge to respond immediately may differentiate these tasks on this measurement of impulsive action and perhaps alter the role of the mPFC in controlling this aspect of impulsivity.

Considering that the IGT was developed in part to aid in characterizing cognitive deficits in patients with damage to the ventromedial prefrontal cortex (Bechara et al. 1994, 1996), it may be surprising that inactivation of the OFC did not alter decision-making. However, studies employing a variant of the IGT in which the chance of winning or losing in each deck are distributed more randomly find that patients with ventromedial prefrontal cortex lesions are not impaired in this form of decision-making (Fellows and Farah 2005). Likewise, rats with bilateral lesions of the OFC also prefer the advantageous options on the rGT (Zeeb and Winstanley 2011). However, if rats received a bilateral OFC lesion prior to training on the rGT, animals required more sessions than control rats to largely prefer the advantageous option (Zeeb and Winstanley 2011). Together with the results from the present study, these findings further support the hypothesis that the OFC may have an important role in learning about probabilistic schedules of reinforcement involving both rewards and losses in order to establish an optimal decisionmaking strategy, rather than promoting choice of the advantageous options once the contingencies have been learned (Zeeb and Winstanley 2011, 2013). 
Many imaging studies indicate that the ACC plays an important role during IGT performance (Ernst et al. 2002; Bolla et al. 2003; Li et al. 2010) and in other tasks that test probabilistic decision-making involving gains and losses (Rogers et al. 2004). Although damage to the ACC increased choice of the disadvantageous options on the IGT (Naccache et al. 2005; Njomboro et al. 2012), acute inactivation of the ACC did not affect decision-making in the present study. Our results are in line with a previous study using a different rat analogue of the IGT that reported null effects of ACC lesions (Rivalan et al. 2011). Interestingly, neither human nor animal data support a role of the ACC in simple probabilistic or delay-based decision-making (Rogers et al. 1999; Cardinal et al. 2001). In contrast, pre-clinical studies have demonstrated that inactivation or lesion of the ACC, but not the PrL or IL cortices, biased animals toward options associated with less physical effort, but also less reward (Walton et al. 2003, 2009; Schweimer and Hauber 2005; Rudebeck et al. 2006; Floresco and Ghods-Sharifi 2007; Holec et al. 2014), and neurons in the ACC encode this type of decision-making (Hillman and Bilkey 2010). Likewise, the ACC is also important for guiding decisions based on cognitive effort and conflict resolution in human subjects (Botvinick et al. 1999; Barch et al. 2001; Naccache et al. 2005; Behrens et al. 2007).

It could be argued that the first few trials of the IGT involve a form of cognitive conflict resolution, as subjects are experiencing both gains and losses and must determine which options lead to greater long-term gains. If this is the case, then it could be hypothesized that subjects could improve their performance on the IGT with additional training and exposure to the repeated outcome contingencies. Indeed, rats with pretraining lesions to the ACC required more sessions to perform similarly to control rats on a rat IGT (Rivalan et al. 2011). Furthermore, recordings from neurons in the ACC while animals are performing a decision-making task have demonstrated that when the contingencies of the task change, activity of the neurons within the ACC also change (Lapish et al. 2008), suggesting that this region is highly important during learning in decision-making tasks. Together with the findings from the present study, these results suggest that, similar to the OFC, the ACC may be more important when subjects are learning to balance both potential gains and losses in order to reach the optimal decision. However, once a strategy has been established on the IGT or rGT, the ACC may have a smaller role in maintaining the chosen strategy.

Within the mPFC, DA D2 receptors are thought to play a role in decision-making when subjects are required to avoid aversive stimuli (Floresco and Magyar 2006). Systemic administration of eticlopride, a DA D2 receptor antagonist, increased choice of the optimal option, P2 (Zeeb et al. 2009; Zeeb et al. 2013; but see Paine et al. 2013). Likewise, eticlopride decreased choice of the large reward option associated with greater uncertainty in a probability discounting task (St Onge and Floresco 2009). Theoretically, blockade of prefrontal DA D2 receptors could lead to a more DA D1 receptor dependent state which would produce a more stable network (Seamans et al. 2001; Seamans and Yang 2004; Trantham-Davidson et al. 2004), possibly promoting choice of the most favoured option, P2 in the rGT. However, infusion of eticlopride into any one cortical region tested did not alter decision-making preferences on the rGT. Perhaps, the dose of eticlopride used in the present study was too low to significantly alter neural activity in these regions. Yet, the doses of eticlopride used here were identical to those that significantly affected cognitive processing when infused into the frontal cortex (for example, Zeeb et al. 2010). Alternatively, localized blockade of DA D2 receptors in a single sub-region may not be sufficient to alter decision-making preferences, or the effects observed after systemic eticlopride could reflect changes in dopamine signaling in other regions, such as the basolateral amygdala which is also implicated in maintaining optimal choice (Bechara et al. 1999; Zeeb and Winstanley 2011).

In summary, increased disadvantageous decision-making was observed in well-trained rats following inactivation of two distinct sub-regions of the mPFC: the IL and PrL cortices. These data help to further elucidate the complex role of various regions of the PFC in promoting choice of advantageous options on a translational decision-making task, the rGT. These results also highlight the importance of pre-clinical studies to precisely determine the role of specific cortical regions during cost-benefit decision-making.

Acknowledgments This work was supported by an operating grant awarded to CAW from the Canadian Institutes for Health Research (CIHR) and by National Institute on Drug Abuse Grant R01 DA022628 (LJMJV). CAW also receives salary support through the Michael Smith Foundation for Health Research and the CIHR New Investigator Award program. FDZ currently receives salary support through the CIHR Postdoctoral Fellowship. We thank Suzanne Lemstra and Ruth Damsteegt for practical assistance. CAW has previously consulted for Shire, and FDZ has previously consulted for Intervivo Solutions, Inc, both on unrelated matters. The authors declare no competing financial interests.

\section{References}

Baarendse PJJ, Vanderschuren LJMJ (2012) Dissociable effects of monoamine reuptake inhibitors on distinct forms of impulsive behavior in rats. Psychopharmacology (Berl) 219:313-326. doi:10.1007/ s00213-011-2576-x

Baarendse PJJ, Winstanley CA, Vanderschuren LJMJ (2013) Simultaneous blockade of dopamine and noradrenaline reuptake promotes disadvantageous decision making in a rat gambling task. Psychopharmacology (Berl) 225:719-731. doi:10.1007/s00213012-2857-z

Barch DM, Braver TS, Akbudak E et al (2001) Anterior cingulate cortex and response conflict: effects of response modality and processing domain. Cereb Cortex 11:837-848 
Bechara A, Damasio AR, Damasio H, Anderson SW (1994) Insensitivity to future consequences following damage to human prefrontal cortex. Cognition 50:7-15

Bechara A, Tranel D, Damasio H, Damasio AR (1996) Failure to respond autonomically to anticipated future outcomes following damage to prefrontal cortex. Cereb Cortex 6:215-225. doi:10.1093/cercor/6.2. 215

Bechara A, Damasio H, Damasio AR, Lee GP (1999) Different contributions of the human amygdala and ventromedial prefrontal cortex to decision-making. J Neurosci 19:5473-5481

Bechara A, Tranel D, Damasio H (2000) Characterization of the decisionmaking deficit of patients with ventromedial prefrontal cortex lesions. Brain 123(Pt 11):2189-2202

Bechara A, Dolan S, Denburg N et al (2001) Decision-making deficits, linked to a dysfunctional ventromedial prefrontal cortex, revealed in alcohol and stimulant abusers. Neuropsychologia 39:376-389

Behrens TEJ, Woolrich MW, Walton ME, Rushworth MFS (2007) Learning the value of information in an uncertain world. Nat Neurosci 10:1214-1221. doi:10.1038/nn1954

Bolla KI, Eldreth DA, London ED et al (2003) Orbitofrontal cortex dysfunction in abstinent cocaine abusers performing a decision-making task. Neuroimage 19:1085-1094

Botvinick M, Nystrom LE, Fissell K et al (1999) Conflict monitoring versus selection-for-action in anterior cingulate cortex. Nature 402: 179-181. doi:10.1038/46035

Boulougouris V, Dalley JW, Robbins TW (2007) Effects of orbitofrontal, infralimbic and prelimbic cortical lesions on serial spatial reversal learning in the rat. Behav Brain Res 179:219-228. doi:10.1016/j. bbr.2007.02.005

Cardinal RN (2006) Neural systems implicated in delayed and probabilistic reinforcement. Neural Netw Off J Int Neural Netw Soc 19: 1277-1301. doi:10.1016/j.neunet.2006.03.004

Cardinal RN, Pennicott DR, Sugathapala CL et al (2001) Impulsive choice induced in rats by lesions of the nucleus accumbens core. Science 292:2499-2501. doi:10.1126/science.1060818

Cella M, Dymond S, Cooper A (2010) Impaired flexible decision-making in major depressive disorder. J Affect Disord 124:207-210. doi:10. 1016/j.jad.2009.11.013

Chudasama Y, Passetti F, Rhodes SE, Lopian D, Desai A, Robbins TW (2003) Dissociable aspects of performance on the 5-choice serial reaction time task following lesions of the dorsal anterior cingulate, infralimbic and orbitofrontal cortex in the rat: differential effects on selectivity, impulsivity and compulsivity. Behav Brain Res 146(1-2):105-19

Clark L, Manes F, Antoun N et al (2003) The contributions of lesion laterality and lesion volume to decision-making impairment following frontal lobe damage. Neuropsychologia 41:1474-1483

Connolly NP, Kim JS, Tunstall BJ, Kearns DN (2015) A test of stress, cues, and re-exposure to large wins as potential reinstaters of suboptimal decision making in rats. Front Psychol 6:394. doi:10.3389/ fpsyg.2015.00394

Dalley JW, Cardinal RN, Robbins TW (2004) Prefrontal executive and cognitive functions in rodents: neural and neurochemical substrates. Neurosci Biobehav Rev 28:771-784. doi:10.1016/j.neubiorev.2004. 09.006

Eagle DM, Baunez C, Hutcheson DM, Lehmann O, Shah AP, Robbins TW (2008) Stop-signal reaction-time task performance: role of prefrontal cortex and subthalamic nucleus. Cereb Cortex 18(1):178188

Ernst M, Bolla K, Mouratidis M et al (2002) Decision-making in a risktaking task: a PET study. Neuropsychopharmacology 26:682-691. doi:10.1016/S0893-133X(01)00414-6

Fellows LK, Farah MJ (2005) Different underlying impairments in decision-making following ventromedial and dorsolateral frontal lobe damage in humans. Cereb Cortex 15:58-63. doi:10.1093/ cercor/bhh108
Fitoussi A, Le Moine C, De Deurwaerdère P et al (2014) Prefrontosubcortical imbalance characterizes poor decision-making: neurochemical and neural functional evidences in rats. Brain Struct Funct. doi:10.1007/s00429-014-0868-8

Floresco SB, Ghods-Sharifi S (2007) Amygdala-prefrontal cortical circuitry regulates effort-based decision making. Cereb Cortex 17: 251-260. doi:10.1093/cercor/bhj143

Floresco SB, Magyar O (2006) Mesocortical dopamine modulation of executive functions: beyond working memory. Psychopharmacology (Berl) 188:567-585. doi:10.1007/s00213006-0404-5

Goudriaan AE, Oosterlaan J, de Beurs E, van den Brink W (2005) Decision making in pathological gambling: a comparison between pathological gamblers, alcohol dependents, persons with Tourette syndrome, and normal controls. Brain Res Cogn Brain Res 23: 137-151. doi:10.1016/j.cogbrainres.2005.01.017

Hewig J, Straube T, Trippe RH et al (2009) Decision-making under risk: an fMRI study. J Cogn Neurosci 21:1642-1652. doi:10.1162/jocn. 2009.21112

Hillman KL, Bilkey DK (2010) Neurons in the rat anterior cingulate cortex dynamically encode cost-benefit in a spatial decisionmaking task. J Neurosci 30:7705-7713. doi:10.1523/ JNEUROSCI.1273-10.2010

Holec V, Pirot HL, Euston DR (2014) Not all effort is equal: the role of the anterior cingulate cortex in different forms of effort-reward decisions. Front Behav Neurosci 8:12. doi:10.3389/fnbeh.2014.00012

Hoover WB, Vertes RP (2007) Anatomical analysis of afferent projections to the medial prefrontal cortex in the rat. Brain Struct Funct 212:149-179. doi:10.1007/s00429-007-0150-4

Lapish CC, Durstewitz D, Chandler LJ, Seamans JK (2008) Successful choice behavior is associated with distinct and coherent network states in anterior cingulate cortex. Proc Natl Acad Sci U S A 105: 11963-11968. doi:10.1073/pnas.0804045105

Lawrence NS, Jollant F, O'Daly O et al (2009) Distinct roles of prefrontal cortical subregions in the Iowa Gambling Task. Cereb Cortex 19: 1134-1143. doi:10.1093/cercor/bhn154

Li X, Lu Z-L, D'Argembeau A et al (2010) The Iowa Gambling Task in fMRI images. Hum Brain Mapp 31:410-423. doi:10.1002/hbm. 20875

Manes F, Sahakian B, Clark L et al (2002) Decision-making processes following damage to the prefrontal cortex. Brain 125:624-639

McDonald J (2009) Handbook of biological statistics, 2nd edn. Sparky House Publishing, Baltimore

Muir JL, Everitt BJ, Robbins TW (1996) The cerebral cortex of the rat and visual attentional function: dissociable effects of mediofrontal, cingulate, anterior dorsolateral, and parietal cortex lesions on a fivechoice serial reaction time task. Cerebral Cortex 6(3):470-481

Murphy ER, Fernando AB, Urcelay GP, Robinson ES, Mar AC, Theobald DE, Dalley JW, Robbins TW (2012) Impulsive behaviour induced by both NMDA receptor antagonism and GABAA receptor activation in rat ventromedial prefrontal cortex. Psychopharmacology (Berl). 219(2):401-410

Naccache L, Dehaene S, Cohen L et al (2005) Effortless control: executive attention and conscious feeling of mental effort are dissociable. Neuropsychologia 43:1318-1328. doi:10.1016/j.neuropsychologia. 2004.11.024

Njomboro P, Deb S, Humphreys GW (2012) Apathy and executive functions: insights from brain damage involving the anterior cingulate cortex. BMJ Case Rep. doi:10.1136/bcr-02-2012-5934

Paine TA, Asinof SK, Diehl GW et al (2013) Medial prefrontal cortex lesions impair decision-making on a rodent gambling task: reversal by D1 receptor antagonist administration. Behav Brain Res 243: 247-254. doi:10.1016/j.bbr.2013.01.018

Paine TA, O'Hara A, Plaut B, Lowes DC (2014) Effects of disrupting medial prefrontal cortex GABA transmission on decision-making in 
a rodent gambling task. Psychopharmacology (Berl). doi:10.1007/ s00213-014-3816-7

Pattij T, Vanderschuren LJMJ (2008) The neuropharmacology of impulsive behaviour. Trends Pharmacol Sci 29:192-199. doi:10.1016/j. tips.2008.01.002

Paxinos G, Watson C (1998) The rat brain in stereotaxic co-ordinates. Academic, Sydney

Ragozzino ME (2007) The contribution of the medial prefrontal cortex, orbitofrontal cortex, and dorsomedial striatum to behavioral flexibility. Ann N Y Acad Sci 1121:355-375. doi:10.1196/annals.1401.013

Rivalan M, Coutureau E, Fitoussi A, Dellu-Hagedorn F (2011) Interindividual decision-making differences in the effects of cingulate, orbitofrontal, and prelimbic cortex lesions in a rat gambling task. Front Behav Neurosci 5:22. doi:10.3389/fnbeh.2011.00022

Robbins TW (2002) The 5-choice serial reaction time task: behavioural pharmacology and functional neurochemistry. Psychopharmacology (Berl) 163:362-380. doi:10.1007/s00213-002-1154-7

Rogers RD, Owen AM, Middleton HC et al (1999) Choosing between small, likely rewards and large, unlikely rewards activates inferior and orbital prefrontal cortex. J Neurosci 19:9029-9038

Rogers RD, Ramnani N, Mackay C et al (2004) Distinct portions of anterior cingulate cortex and medial prefrontal cortex are activated by reward processing in separable phases of decision-making cognition. Biol Psychiatry 55:594-602. doi:10.1016/j.biopsych.2003.11.012

Rudebeck PH, Walton ME, Smyth AN et al (2006) Separate neural pathways process different decision costs. Nat Neurosci 9:1161-1168. doi:10.1038/nn1756

Schweimer J, Hauber W (2005) Involvement of the rat anterior cingulate cortex in control of instrumental responses guided by reward expectancy. Learn Mem 12:334-342. doi:10.1101/lm.90605

Seamans JK, Yang CR (2004) The principal features and mechanisms of dopamine modulation in the prefrontal cortex. Prog Neurobiol 74:158. doi:10.1016/j.pneurobio.2004.05.006

Seamans JK, Gorelova N, Durstewitz D, Yang CR (2001) Bidirectional dopamine modulation of GABAergic inhibition in prefrontal cortical pyramidal neurons. J Neurosci 21:3628-3638

Seamans JK, Lapish CC, Durstewitz D (2008) Comparing the prefrontal cortex of rats and primates: insights from electrophysiology. Neurotox Res 14:249-262. doi:10.1007/BF03033814

Shurman B, Horan WP, Nuechterlein KH (2005) Schizophrenia patients demonstrate a distinctive pattern of decision-making impairment on the Iowa Gambling Task. Schizophr Res 72:215-224. doi:10.1016/j. schres.2004.03.020

Smoski MJ, Lynch TR, Rosenthal MZ et al (2008) Decision-making and risk aversion among depressive adults. J Behav Ther Exp Psychiatry 39:567-576. doi:10.1016/j.jbtep.2008.01.004

St Onge JR, Floresco SB (2009) Dopaminergic modulation of risk-based decision making. Neuropsychopharmacology 34:681-697. doi:10. 1038/npp.2008.121

St Onge JR, Floresco SB (2010) Prefrontal cortical contribution to riskbased decision making. Cereb Cortex 20:1816-1828. doi:10.1093/ cercor/bhp250

Trantham-Davidson H, Neely LC, Lavin A, Seamans JK (2004) Mechanisms underlying differential D1 versus D2 dopamine receptor regulation of inhibition in prefrontal cortex. J Neurosci 24:10652-10659. doi:10.1523/JNEUROSCI.3179-04.2004

Tsutsui-Kimura I, Yoshida T, Ohmura Y, Izumi T, Yoshioka M (2014) Milnacipran remediates impulsive deficits in rats with lesions of the ventromedial prefrontal cortex. Int J Neuropsychopharmacol 8(5). doi:10.1093/ijnp/pyu083

Uylings HBM, Groenewegen HJ, Kolb B (2003) Do rats have a prefrontal cortex? Behav Brain Res 146:3-17

Veeneman MMJ, Broekhoven MH, Damsteegt R, Vanderschuren LJMJ (2012) Distinct contributions of dopamine in the dorsolateral striatum and nucleus accumbens shell to the reinforcing properties of cocaine. Neuropsychopharmacology 37:487-498. doi:10.1038/ npp.2011.209

Vertes RP (2004) Differential projections of the infralimbic and prelimbic cortex in the rat. Synapse 51:32-58. doi:10.1002/ syn. 10279

Walton ME, Bannerman DM, Alterescu K, Rushworth MFS (2003) Functional specialization within medial frontal cortex of the anterior cingulate for evaluating effort-related decisions. J Neurosci 23: 6475-6479

Walton ME, Groves J, Jennings KA et al (2009) Comparing the role of the anterior cingulate cortex and 6-hydroxydopamine nucleus accumbens lesions on operant effort-based decision making. Eur J Neurosci 29:1678-1691. doi:10.1111/j.1460-9568.2009. 06726.x

Windmann S, Kirsch P, Mier D et al (2006) On framing effects in decision making: linking lateral versus medial orbitofrontal cortex activation to choice outcome processing. J Cogn Neurosci 18:1198-1211. doi: 10.1162/jocn.2006.18.7.1198

Zeeb FD, Winstanley CA (2011) Lesions of the basolateral amygdala and orbitofrontal cortex differentially affect acquisition and performance of a rodent gambling task. J Neurosci 31(6):2197-2204. doi:10. 1523/JNEUROSCI.5597-10.2011

Zeeb FD, Winstanley CA (2013) Functional disconnection of the orbitofrontal cortex and basolateral amygdala impairs acquisition of a rat gambling task and disrupts animals' ability to alter decision-making behavior after reinforcer devaluation. J Neurosci 33:6434-6443. doi:10.1523/JNEUROSCI.3971-12. 2013

Zeeb FD, Robbins TW, Winstanley CA (2009) Serotonergic and dopaminergic modulation of gambling behavior as assessed using a novel rat gambling task. Neuropsychopharmacology 34:2329-2343. doi: $10.1038 /$ npp. 2009.62

Zeeb FD, Floresco SB, Winstanley CA (2010) Contributions of the orbitofrontal cortex to impulsive choice: interactions with basal levels of impulsivity, dopamine signalling, and reward-related cues. Psychopharmacology (Berl) 211(1):87-98. doi:10.1007/s00213010-1871-2

Zeeb FD, Wong AC, Winstanley CA (2013) Differential effects of environmental enrichment, social-housing, and isolation-rearing on a rat gambling task: dissociations between impulsive action and risky decision-making. Psychopharmacology (Berl) 225:381-395. doi: 10.1007/s00213-012-2822-x 